\title{
Development of a novel 96-microwell assay with high throughput for determination of olmesartan medoxomil in its tablets
}

\author{
Ibrahim A Darwish*, Tanveer A Wani, Nasr Y Khalil, Abdul-Aziz Al-Shaikh and Najm Al-Morshadi
}

\begin{abstract}
A novel 96-microwell-based spectrophotometric assay has been developed and validated for determination of olmesartan medoxomil (OLM) in tablets. The formation of a colored charge-transfer (CT) complex between OLM as a n-electron donor and 2, 5-dichloro-3, 6-dihydroxy-1, 4-benzoquinone (p-chloranilic acid, pCA) as a r-electron acceptor was investigated, for the first time, and employed as a basis in the development of the proposed assay. The proposed assay was carried out in 96-microwell plates. The absorbance of the colored-CT complex was measured at $490 \mathrm{~nm}$ by microwell-plate absorbance reader. The optimum conditions of the reaction and the analytical procedures of the assay were established. Under the optimum conditions, linear relationship with good correlation coefficient was found between the absorbance and the concentration of OLM in the range of 1-200 $\mu \mathrm{g}$ $\mathrm{ml}^{-1}$. The limits of detection and quantitation were 0.3 and $1 \mathrm{\mu g} \mathrm{ml}^{-1}$, respectively. No interference was observed from the additives that are present in the pharmaceutical formulation or from hydrochlorothiazide and amlodipine that are co-formulated with OLM in some formulations. The assay was successfully applied to the analysis of OLM in tablets with good accuracy and precision. The assay described herein has great practical value in the routine analysis of OLM in quality control laboratories, as it has high throughput property, consumes minimum volume of organic solvent thus it offers the reduction in the exposures of the analysts to the toxic effects of organic solvents, and reduction in the analysis cost by 50-fold. Although the proposed assay was validated for OLM, however, the same methodology could be used for any electron-donating analyte for which a CT reaction can be performed.
\end{abstract}

Keywords: Olmesartan medoxomil, Charge-transfer reaction, Spectrophotometry, Microwell-based assay, High throughput analysis

\section{Background}

Olmesartan medoxomil (OLM); (5-methyl-2-oxo- $2 H$ 1,3-dioxol-4-yl)methyl 4-(2-hydroxy-propan-2-yl)-2-propyl-1-(\{4-[2-(2H-1,2,3,4-tetrazol-5-yl)phenyl]phenyl $\}$ methyl)-1H-imidazole-5-carboxylate (Figure 1), is the newest member of non-peptide angiotensin II receptor antagonists used worldwide in the treatment of hypertension. It is an ester prodrug, which is completely and rapidly hydrolyzed to the active acid form, olmesartan. OLM exerts its action mainly via a selective blockade action on AT1 receptors and the consequent reduced pressor effect of angiotensin II [1,2]. OLM may be used alone or in combination with other antihypertensive

\footnotetext{
* Correspondence: idarwish@ksu.edu.sa

Department of Pharmaceutical Chemistry, College of Pharmacy, King Saud University, P.O. Box 2457, Riyadh 11451, Saudi Arabia
}

agents. The FDA has determined that the benefits of OLM continue to outweigh its potential risks when used for the treatment of patients with high blood pressure according to the drug label. OLM is also used by some people who are treated with an unproven therapy for autoimmune diseases known as the Marshall Protocol.

OLM has not yet been officially described in any pharmacopoeia. A literature survey revealed that several analytical methods were reported for its determination. These methods include high-performance thin-layer chromatography [3], liquid chromatography [3-9], and capillary zone electrophoresis [10]. These methods were not simple to perform, time-consuming, and utilize expensive instruments that are not available in most quality control laboratories. In general, spectrophotometry is the most widely used technique in pharmaceutical
(C) ChemistryCentral

(C) 2010 Darwish et al; licensee Springer. This is an Open Access article distributed under the terms of the Creative Commons Attribution License (http://creativecommons.org/licenses/by/2.0), which permits unrestricted use, distribution, and reproduction in any medium, provided the original work is properly cited. 


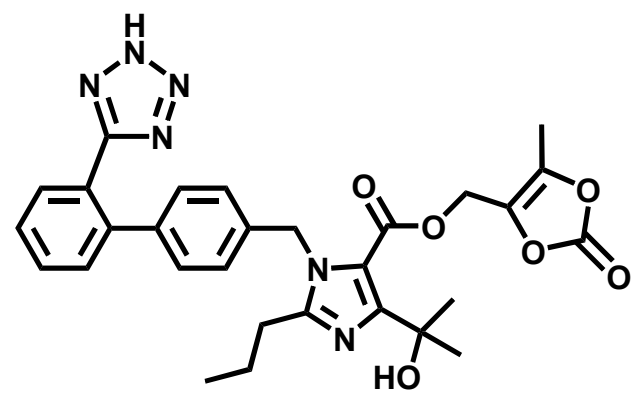

Olmesartan medoxomil (OLM)<smiles>NS(=O)(=O)c1cc2c(cc1Cl)NCNS2(=O)=O</smiles>

Hydrochlorothiazide<smiles>CCOC(=O)C1=C(COCCN)NC(C)=C(C(=O)OC)C1c1ccccc1Cl</smiles>

Amlodipine besylate

Figure 1 The chemical structure of olmesartan medoxomil (OLM) and the co-formulated drugs.

analysis because of its inherent simplicity and wide availability in most quality control laboratories [11]. However, few spectrophotometric methods have been reported for the analysis of OLM in its pharmaceutical tablets [12-15]. Unfortunately, these methods suffer from major drawbacks such as decreased selectivity due to measuring the native light absorption of OLM in the blue-shifted ultraviolet region, which might be subjected to interferences [12-14]. Besides the tedious liquid-liquid extraction procedures using large volumes of organic solvents in the methods based on formation of ion-pair associates [15]. Therefore, the development of a new alternative spectrophotometric method for determination of OLM in pharmaceutical formulations is very essential.

The molecular interactions between the electrondonating pharmaceutical compounds and electronaccepting reagents are generally associated with the formation of intensely colored CT complexes, which usually absorb radiations in the visible region. The rapid formation of these complexes leads to their widespread utility in the development of visible spectrophotometric methods for analysis of many pharmaceutical compounds [16-20]. Literature survey revealed that the CT reaction of OLM has not been investigated yet. This fact promoted our interest in employment of the CT-reaction as a basis for the development of a new spectrophotometric method for determination of OLM. However, all the conventional CT-based spectrophotometric methods that have been reported so far are not automated and consequently their throughput is low, thus their applications in pharmaceutical quality control laboratories are limited. Moreover, these methods suffer from the consumption of large volumes of organic solvents, which leads to high analysis cost, and more importantly, the incidence of exposure of the analysts to the toxic effects of the organic solvents [21-25].

For these reasons, the present study is devoted to investigate the CT reaction of OLM, and its employment in the development of a novel non-conventional spectrophotometric assay with high analysis throughput and can reduce the consumption of organic solvents in the determination of OLM in tablets.

\section{Experimental}

Apparatus

Microwell-plate absorbance reader (ELx 808, Bio-Tek Instruments Inc. Winooski, USA) was used for all the measurements in 96-microwell plates. UV-1601 PC (Shimadzu, Kyoto, Japan) ultraviolet-visible spectrophotometer with matched $1 \mathrm{~cm}$ quartz cells was used for recording the absorption spectra. 96-Microwell plates were a product of Corning/Costar Inc. (Cambridge, USA). Finnpipette adjustable 8-channel-pipette was obtained from Sigma Chemical Co. (St. Louis, MO, USA).

\section{Chemicals and tablets}

OLM was obtained from AK Scientific Inc. (California, USA). Amlodipine besylate and hydrochlorothiazide were obtained from Sigma Chemical Co. (St. Louis, USA). pCA (BDH Chemicals, Poole, UK) was $0.5 \%(\mathrm{w} / \mathrm{v})$ in acetonitrile and it was prepared fresh daily. Olmetec ${ }^{\circledR}$ tablets (SAJA Pharmaceuticals, Jeddah, Saudi Arabia) labeled to contain $20 \mathrm{mg}$ OLM were obtained from the local market. 
Preparation of standard and tablet solutions

Preparation of stock standard solutions

Into a 5 - $\mathrm{ml}$ calibrated flask, $10 \mathrm{mg}$ of OLM was accurately weighed, dissolved in $2 \mathrm{ml}$ methanol, and completed to volume with the same solvent. This stock solution was diluted with methanol to obtain the suitable concentrations that lie in the linear range of the assay.

\section{Preparation of tablet sample solutions}

Twenty tablets were weighed and finely powdered. A quantity of the powder equivalent to $20 \mathrm{mg}$ of OLM was transferred into a $10-\mathrm{ml}$ calibrated flask, dissolved in $4 \mathrm{ml}$ methanol, swirled and sonicated for $5 \mathrm{~min}$, completed to volume with the methanol, shaken well for 15 min, and filtered. The first portion of the filtrate was rejected, and a measured volume of the filtrate was diluted quantitatively with methanol to yield the suitable concentrations that lie in the linear range of the assay.

\section{General analytical procedure}

Accurately measured aliquots $(100 \mu \mathrm{l})$ of the standard or sample solution containing varying amounts of OLM (1-200 $\mathrm{g} \mathrm{m} \mathrm{m}^{-1}$ ) were transferred into wells of 96-microwell assay plates. One hundred microliters of pCA solution $(0.5 \%, \mathrm{w} / \mathrm{v})$ was added, and the reaction was allowed to proceed at room temperature $\left(25 \pm 1^{\circ} \mathrm{C}\right)$ for $5 \mathrm{~min}$. The absorbances of the resulting solutions were measured at $490 \mathrm{~nm}$ by the microwell-plate reader. Blank wells were treated similarly except $100 \mu \mathrm{l}$ of methanol was used instead of sample, and the absorbances of the blank wells were subtracted from those of the other wells.

\section{Determination of molar ratio}

The Job's method of continuous variation [26] was employed. Master equimolar solutions $\left(2 \times 10^{-3} \mathrm{M}\right)$ of each of OLM and pCA were prepared. Series of $200 \mu \mathrm{l}$ portions of the master solutions of OLM with pCA were made up comprising different complementary ratios $(0: 10,1: 9, \ldots \ldots . . ., 9: 1,10: 0$, inclusive) in each well of the 96-microwell assay plate. The reaction was allowed to proceed at room temperature $\left(25 \pm 1^{\circ} \mathrm{C}\right)$ for $5 \mathrm{~min}$. The absorbances of the developed colors were measured at $490 \mathrm{~nm}$ by the microwell-plate reader against blank wells treated similarly except methanol was used instead of OLM sample. The measured absorbances were plotted as a function of OLM mole fraction. The generated plot was used for determination the molar ration of OLM:pCA.

\section{Molecular modeling for the CT complex of OLM with pCA}

The molecular modeling for the CT complex was performed by using CS Chem3D Ultra, version 9 (Cambridge Soft Corporation, Cambridge, MA, USA) implemented with molecular orbital computations software (MOPAC), and molecular dynamics computations software (MM2).

\section{Results and discussion}

\section{Strategy for assay development and its design}

In the present study, OLM was selected based on its therapeutic importance, clinical success, and the expected electron-donating ability. Previous studies involving CT reactions with polyhalo-/polycyanoquinone electron $n$-acceptors revealed that $\mathrm{pCA}$ is one of the most efficient reagents in terms of its reactivity [18,27]. Furthermore, its CT reaction with electron-donating analytes is instantaneous $[18,19]$. For these reasons, pCA was used as electron acceptor in the development of the proposed assay. The proposed assay was designed to employ 96-microwell assay plate as the CT reaction was carried out in microwells of the assay plate $(200-\mu \mathrm{l}$ reaction volume). The solutions were dispensed by 8-channel pipette, and the absorbances of the colored CT complex were measured by 96-microwell-plate absorbance reader. The 96-microwell design of the proposed assay was based on the previous success of Darwish et al [28] in the utility of this design for determination of some other pharmaceuticals.

\section{Reaction and spectral characteristics}

The interaction of OLM with pCA was allowed to proceed at room temperature, and the absorption spectrum of the produced chromogen was recorded on an ultraviolet-visible spectrophotometer (UV-1601 PC (Shimadzu, Kyoto, Japan). OLM gave red colored chromogen showing absorption maximum at $520 \mathrm{~nm}$. This band was attributed to the formation of the radical anion $\mathrm{pCA}^{-}$[29], which was probably formed by the dissociation of an original donor-acceptor (D-A) complex:

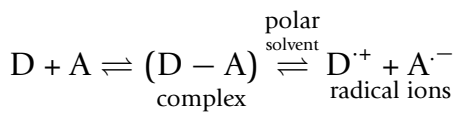

pCA exists in three ionic forms, the neutral yelloworange $\mathrm{H}_{2} \mathrm{~A}$ at very low $\mathrm{pH}$, the dark purple $\mathrm{HA}^{-}$which is stable at $\mathrm{pH}=3$ and a pale violet $\mathrm{A}^{2-}$, which is stable at high $\mathrm{pH}$; these transformations are illustrated in the following scheme:

$$
\begin{aligned}
& \mathrm{H}_{2} \mathrm{~A} \rightleftharpoons \mathrm{H}^{+}+\mathrm{HA}^{-} \text {(violet), } \\
& \mathrm{HA}^{-} \rightleftharpoons \mathrm{H}^{+}+\mathrm{A}^{2-} \text { (colorless) } .
\end{aligned}
$$

Since the interaction of OLM with pCA in acetonitrile gave a violet product, it might be concluded that $\mathrm{HA}^{-}$ was the form of $\mathrm{pCA}$ involved in the reaction described herein.

\section{Optimization of experimental conditions}

The optimization of experimental conditions affecting the reaction in the 96 -well format was investigated by 
altering each reaction variable in a turn while keeping the others constant. Although, the OLM-pCA complex exhibited maximum absorption peak at $520 \mathrm{~nm}$, however the measurements were carried out at $490 \mathrm{~nm}$, as this is the closest wavelength filter available in the plate reader. The results of variations in the pCA concentrations indicated that $100 \mu \mathrm{l}$ of $0.5 \%(\mathrm{w} / \mathrm{v})$ was the optimum pCA concentration, as this concentration gave the highest absorbances. Previous studies [30] demonstrated that the interaction of electron-donors with pCA in polar solvents (e.g. methanol and acetonitrile) produces CT complexes with molar absorptivity values higher than those produced in non-polar solvents (e.g. chloroform). Different polar solvents were tested as a solvent for preparing $\mathrm{pCA}$; these solvents were methanol, ethanol, 1-propanol, 1-butanol, and acetonitrile. Acetonitrile offered the highest sensitivity, therefore it was selected. The optimum reaction time was determined by monitoring the color development in the microwells at room temperature $\left(25 \pm 1^{\circ} \mathrm{C}\right)$. Complete color development was attained instantaneously, however for higher precision readings, the reaction was allowed to proceed for 5 $\mathrm{min}$. The developed color remained stable at room temperature for at least a further $30 \mathrm{~min}$. A summary for the optimum conditions is given in Table 1.

\section{Molar ratio of the reaction, molecular modeling, and proposing the site of interaction}

Job's method of continuous variation [26] was used for determining the molar ratio of OLM to pCA. From the obtained Job's plot, it was concluded that the OLM:pCA ratio is $1: 1$. This indicated that only one site of interaction was involved in the formation of the colored CT complex in spite of the presence of more than one possible electron-donating sites in OLM structure (e.g. $\mathrm{OH}$ of the hydroxypropane-2-yl group and the nitrogen atoms of the tetrazole ring). For investigating the site of interaction and postulate the reaction mechanism, modeling for the CT complex was performed. OLM and pCA were energy-minimized alone and both together. It was found that the electron densities in OLM molecule that are located on the oxygen atom of the $\mathrm{OH}$ of the

Table 1 Optimum conditions for the charge-transfer reaction of OLM with PCA

\begin{tabular}{lcc}
\hline Condition & Studied range & Optimum \\
\hline pCA conc. $(\%, w / v)$ & $0.1-2$ & 0.5 \\
Solvent & Different $^{\mathrm{a}}$ & Acetonitrile \\
Reaction time $(\mathrm{min})$ & $0-30$ & 5 \\
Temperature $\left({ }^{\circ} \mathrm{C}\right)$ & $25-60$ & 25 \\
$\lambda_{\max }(\mathrm{nm})$ & $400-600$ & $520^{\mathrm{b}}$
\end{tabular}

a Solvents tested: methanol, ethanol, 1-propanol, 1-butanol, and acetonitrile.

${ }^{\mathrm{b}}$ Measurements were carried out at $490 \mathrm{~nm}$. hydroxypropane-2-yl group, oxygen atom of the ester linkage, and nitrogen atoms of the tetrazole are comparable $(-0.39428,-0.33456$, and -0.33085$)$. As well, it was found that pCA moves toward the tetrazole moiety of OLM to form the CT complex (Figure 2). These facts, taking the molar ratio in account, confirmed that only the tetrazole is involved in the complex formation. The other anticipated centers did not contribute in the CT reaction, probably due the steric hindrance effect of the OLM molecules [31].

\section{Validation of the proposed assay Linearity and sensitivity}

Under the above mentioned optimum reaction conditions, the calibration curve for the analysis of OLM by the proposed assay was constructed by plotting the absorbances as a function of the corresponding concentrations. The regression equation for the results was derived using the least-squares method. Beer's law plot (10-points) was linear in the range of $1-200 \mu \mathrm{g} \mathrm{ml}^{-1}$. The calibration equation was: $\mathrm{A}=-0.0335+0.0827 \mathrm{C}(\mathrm{r}$ $=0.9951$ ), where $\mathrm{A}, \mathrm{C}$ and $\mathrm{r}$ are the absorbance, concentration of OLM and correlation coefficient, respectively. The limits of detection (LOD) and quantitation (LOQ) were determined [32] using the formula: LOD or LOQ $=\kappa \mathrm{SDa} / \mathrm{b}$, where $\kappa=3$ for LOD and 10 for LOQ, SDa is the standard deviation of the intercept, and $b$ is the slope. The LOD and LOQ values were 0.3 and $1 \mu \mathrm{g} \mathrm{ml}{ }^{-}$ ${ }^{1}$, respectively. The quantitative parameters of the proposed assay are given in Table 2.

\section{Accuracy and precision}

Accuracy of the proposed assay was assessed by analytical recovery studies. Recovery was determined by the standard addition method. Known amounts of OLM were added to pre-determined drug-containing tablets, and then determined by the proposed assay. The mean analytical recovery was calculated and found to be 97.6$99.7 \pm 0.5-1.5 \%$ indicating the accuracy of the proposed assay.

The precisions of the proposed assay were determined on samples of drug solutions at three concentration levels for each drug (Table 3) by analyzing 5 replicates of each sample as a batch in a single assay run. The relative standard deviations (RSD) did not exceed $2 \%$ (Table 3) proving the high precision of the assay for the routine application in quality control laboratories.

This high level of precision was attributed to the accuracy of the volumes that have been concomitantly dispensed in the microwells by multi-channel pipettes, and completeness of the reaction the small volume $(200 \mu \mathrm{l})$. Selectivity

The proposed assay has the advantages that the measurements are performed in the visible region, away from the UV-absorbing interfering substances that 


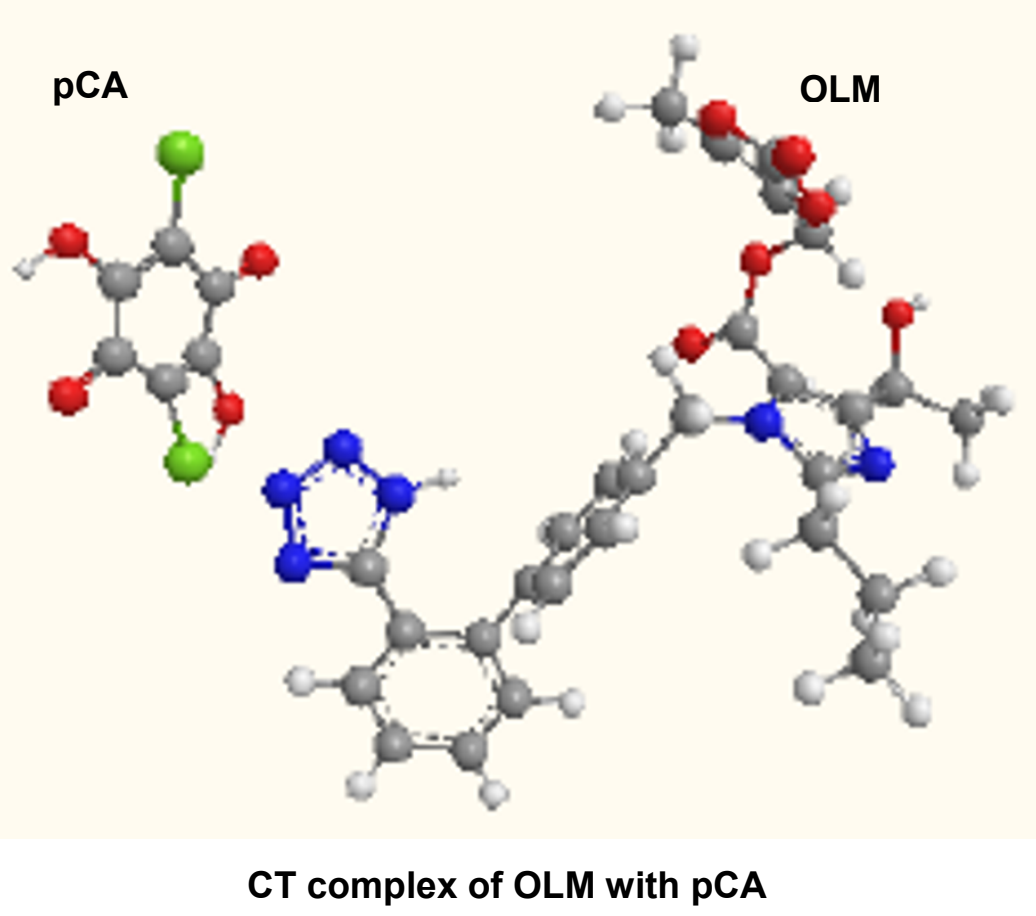

Figure 2 Energy-minimized CT complex of OLM with pCA.

might be co-extracted from dosage forms that contain OLM. The interference from the congenital drugs that is co-formulated with OLM in some dosage forms was studied. These drugs were amlodipine besylate [33-35] hydrochlorothaizde $[35,36]$ and the chemical structures of these drugs are given in Figure 1. Potential interferences of these drugs were studied in a ratio which is normally present in their combined dosage forms. No interferences from these drugs were found with OLM in the proposed assay.

This specificity of the CT reaction for OLM was attributed to its basic character, which allows the CT, rather than hydrochlorothaizde, that does not have sufficient basicity to achieve CT reaction ( $\mathrm{pKa}=7.9)$ [37]. Although, amlodipine has basic character, however it is co-formulated with OLM as besylate salt that did not show any ability for $\mathrm{CT}$ reaction with $\mathrm{pCA}$ under the

Table 2 Quantitative parameters for the analysis of OLM by the proposed assay

\begin{tabular}{ll}
\hline Parameter & Value \\
\hline Range $\left(\mu \mathrm{g} \mathrm{m}^{-1}\right)$ & $1-200$ \\
Intercept & -0.0335 \\
Slope & 0.0827 \\
Correlation coefficient & 0.9951 \\
LOD $\left(\mu \mathrm{g} \mathrm{ml}^{-1}\right)$ & 0.3 \\
LOQ $\left(\mu \mathrm{gl}^{-1}\right)$ & 1 \\
\hline
\end{tabular}

proposed assay conditions. As well, no interference was observed from the excipients with the proposed assay as indicated from the obtained good recovery (mentioned above). The absence of interference from the excipients, even though they contain basic component(s) was attributed to the extraction of the OLM tablets prior to the analysis with methanol in which the excipients do not dissolve.

Application of the proposed assay in the analysis of OLM tablets

The commercially available tablets of OLM (Olmetec ${ }^{\circledR}$ tablets) was subjected to the analysis by the proposed and reported methods [4], and the obtained results were then statistically compared with each other. The mean percentage recovery, relative to the labeled amounts, obtained by the proposed assay was $99.8 \pm 1.24 \%$ (Table 4). Values for $\mathrm{t}$ - and F-tests were calculated and found to be 2.35 and 2.13 , respectively. These calculated $\mathrm{t}$ - and

Table 3 Precision of the proposed assay at different OLM concentrations

\begin{tabular}{|c|c|c|}
\hline \multirow{2}{*}{$\begin{array}{l}\text { Concentration ( } \mu \mathrm{g} \mathrm{ml}^{-} \\
\text {1) }\end{array}$} & \multicolumn{2}{|c|}{ Relative standard deviation } \\
\hline & $\begin{array}{l}\text { Within-assay, } n= \\
5\end{array}$ & $\begin{array}{l}\text { Between-assays, } n= \\
5\end{array}$ \\
\hline 30 & 1.05 & 1.77 \\
\hline 60 & 1.19 & 1.99 \\
\hline 180 & 1.21 & 1.55 \\
\hline
\end{tabular}


Table 4 Analysis of OLM in tablets by the reported and proposed methods

\begin{tabular}{lllll}
\hline Tablet & \multicolumn{2}{l}{ Content $(\% \pm$ SD) } & t-Value $^{\mathbf{b}}$ & F-value $^{\mathbf{b}}$ \\
\cline { 2 - 4 } & $\begin{array}{l}\text { Proposed } \\
\text { method }\end{array}$ & $\begin{array}{l}\text { Reported } \\
\text { method }\end{array}$ & & \\
\hline $\begin{array}{l}\text { Olmetec } \\
\text { tablets }\end{array}$ & $99.8 \pm 1.24$ & $100.2 \pm 0.9$ & 2.35 & 2.13 \\
\hline
\end{tabular}

${ }^{\text {a }}$ Values are mean of five determinations \pm SD.

b The tabulated values at $95 \%$ confidence limit are 2.78 and 6.39 , respectively.

F-values were lower than the tabulated ones (2.78 and 9.39, respectively) indicating that there was no significant differences between the both the proposed and the reported assays at 95\% confidence level in terms their accuracy and precision.

\section{Conclusion}

The present study described the development and validation of a novel microwell-based spectrophotometric assay for the determination of OLM based on its CT reaction with $\mathrm{pCA}$ reagent. In this assay, the $\mathrm{CT}$ reaction was carried out in 96-microwell plates $(200-\mu \mathrm{l}$ reaction volume) instead of the conventional volumetric flasks $(10,000-\mu \mathrm{l}$ volume). The absorbances were measured by microwell-plate reader instead of the conventional spectrophotometer. The assay described herein offered the following advantages:

- Reduction in the consumption of organic solvents in the CT-based spectrophotometric analysis, accordingly reduction in the exposures of the analysts to the toxic effects of organic solvents.

- Reduction in the analysis cost by 50 -folds which can be reflected on the price for the finished dosage forms, thus it can reduce the expenses for the medications.

- Providing a high throughput analytical methodology that can facilitate the processing of large number of samples in a relatively short time. This property was attributed to the use of multi-channel pipettes for efficient dispensing of the solutions, carrying out the analytical reaction in 96-well plates (as reaction vessels), and measuring the color signals in the 96 wells at $\sim 30 \mathrm{sec}-$ onds by the plate reader.

- Although the proposed assay was developed and validated for OLM, however, it is also anticipated that the same methodology could be used for essentially any analyte that can exhibit CT reaction.

\section{Abbreviations}

CT: charge-transfer; OLM: Olmesartan medoxomil; pCA: p-chloranilic acid; LOD: limit of detection; LOQ: limit of quantification; SD: standard deviation; RSD: relative standard deviation;

\section{Acknowledgements}

The authors extend their appreciation to the Deanship of Scientific Research at King Saud University for funding the work through the research group
No. RGP-VPP-065. As well, the authors are grateful to Dr. Hamdy M. AbdelRahman (Department of Pharmaceutical Medicinal Chemistry, Faculty of Pharmacy, Assiut University, Egypt) for performing the molecular modeling.

\section{Authors' contributions}

ID designed the study, participated in the results discussion and prepared the manuscript. TW and NK participated in conducting the assay validation and analysis of dosage forms. AA and NA participated in optimizing of the assay conditions and its validation. All authors read and approved the final manuscript.

\section{Competing interests}

The authors declare that they have no competing interests.

Received: 9 October 2011 Accepted: 3 January 2012

Published: 3 January 2012

\section{References}

1. Burnier M, Brunner HR: Angiotensin II receptor antagonists. Lancet 2000, 355:637-645

2. Aulakh GK, Sodhi RK, Singh M: An update on non-peptide angiotensin receptor antagonists and related RAAS modulators. Life Sci 2007, 81:615-639.

3. Bari PD, Rote AR: RP-LC and HPTLC methods for the determination of olmesartan medoxomil and hydrochlorothiazide in combined tablet dosage forms. Chromatographia 2009, 69:1469-1472.

4. Liu D, Hu P, Matsushima N, Li X, Li L, Jiang J: Quantitative determination of olmesartan in human plasma and urine by liquid chromatography coupled to tandem mass spectrometry. J Chromatogr B Analyt Technol Biomed Life Sci 2007, 856:190-197.

5. Vaidya W, Roy MN, Yetal SM, Joshi SS, Parekh SA: LC-MS-MS Determination of olmesartan in human plasma. Chromatographia 2008, 67:174-150.

6. Liu D, Jiang J, Wang P, Feng S, Hu P: Simultaneous quantitative determination of olmesartan and hydrochlorothiazide in human plasma and urine by liquid chromatography coupled to tandem mass spectrometry. J Chromatogr B Analyt Technol Biomed Life Sci 2010, 878:743-748

7. Sharma RN, Pancholi SS: RP-HPLC-DAD method for determination of olmesartan medoxomil in bulk and tablets exposed to forced conditions. Acta Pharm 2010, 60:13-24.

8. Chouhan KS, Bhure MV, Hemke AT, Gupta KR, Wadodkar SG: Development of RP-HPLC method for estimation of hydrochlorothiazide and olmesartan medoxomil in pharmaceutical formulation. Res J Pharma Biol Chem Sci 2010, 1:78-84

9. Sengupta P, Das A, Pal TK: Stability-indicating RP-HPLC method for simultaneous determination of olmesartan medoxamil and pioglitazone in fixed dose combination tablet dosage form. Asian J Chem 2010, 22:6471-6479.

10. Mustafa C, Sacide A: Development of a CZE method for the determination of olmesartan medoxomil in tablets. Chromatographia 2007, 66:929-933.

11. Gorog S: Ultraviolet-visible spectrophotometry in pharmaceutical analysis Boca Raton, FL: CRC Press; 1995.

12. Celebier M, Altinoz S: Determination of olmesartan medoxomil in tablets by uv-vis spectrophotometry. Pharmazie 2007, 62:419-422.

13. Hemke AT, Bhure MV, Chouhan KS, Gupta KR, Wadodkar SG: UV Spectrophotometric determination of hydrochlorothiazide and olmesartan medoxomil in pharmaceutical formulation. E-Journal of Che 2010, 7:1156-1161.

14. Verma PK, Kamboj VK, Ranjan S: Spectrophotometric estimation of olmesartan medoxomil in tablet dosage form with stability studies. Int J ChemTech Res 2010, 2:1129-1134.

15. Caglar S, Onal A: Two simple and rapid spectrophotometric methods for the determination of a new antihypertensive drug olmesartan in tablets. J Anal Chem 2010, 65:239-243.

16. Darwish IA: Development and validation of spectrophotometric methods for determination of fluoxetine, sertraline, and paroxetine in pharmaceutical dosage forms. J AOAC Int 2005, 88:38-45.

17. Darwish IA: Kinetic spectrophotometric assays for determination of trimetazidine dihydrochloride. Anal Chim Acta 2005, 551:222-231. 
18. Darwish IA: Analytical study for the charge transfer complexes of losartan potassium. Anal Chim Acta 2005, 549:212-220.

19. Darwish IA, Refaat IH: Spectrophotometric analysis of selective serotonin reuptake inhibitors based on formation of charge-transfer complexes with tetracyanoquinodimethane and chloranilic acid. J AOAC Int 2006, 89:326-333.

20. Darwish IA, Abdel-Wadood HA, Abdel-Latif NA: Validated spectrophotometric and fluorimetric assays for analysis of clozapine in tablets and urine. Annali di Chim 2005, 95:345-356.

21. Fidler AT, Baker EL, Letz RE: Neurobehavioural effects of occupational exposure to organic solvents among construction painters. $\mathrm{Br} J$ Ind Med 1987, 44:292-308.

22. Wennborg $H$, Bonde JP, Stenbeck $M$, Olsen J: Adverse reproduction outcomes among employees working in biomedical research laboratories. Scand J Work Environ Health 2002, 28:5-11.

23. Lindbohm ML, Taskinen H, Sallmén M, Hemminki K: Spontaneous abortions among women exposed to organic solvents. Am J Ind Med 1990, 17:449-463.

24. Wennborg H, Bodin L, Vainio H, Axelsson G: Pregnancy outcome of personnel in Swedish biomedical research laboratories. J Occup Environ Med 2000, 42:438-446.

25. Kristensen $P$, Hilt B, Svendsen K, Grimsrud TK: Incidence of lymphohaematopoietic cancer at a university laboratory: a cluster investigation. Eur J Epidemiol 2008, 23:11-15.

26. Job P: Ann. Chem. 16 (1936) 97. Advanced Physicochemical Experiments. 2 edition. Oliner and Boyd, Edinburgh; 1964, 54.

27. Askal HF: Spectrophotometric study of the charge transfer complexes of some pharmaceutical butyrophenones. Talanta 1997, 44:1749-1755.

28. Darwish IA, Mahmoud AM, Al-Majed AR: A novel analytical approach for reducing the consumption of organic solvents in the charge transferbased spectrophotometric analysis: application in the analysis of certain antihypertensive drugs. Acta Pharm 2010, 60:493-501.

29. Taha A, Rücker G: Utility of pi-acceptors in alkaloid assay. Arch Pharm (Weinheim) 1977, 310:485-494.

30. Saleh GA, Askal HF, Darwish IA, El-Shorbagi AN: Spectroscopic analytical study for the charge-transfer complexation of certain cephalosporins with chloranilic acid. Anal Sci 2003, 19:281-287.

31. Foster R: Organic charge-transfer complexes. London, New York, Academic Press; 1969, 470

32. United States Pharmacopeial: The United States Pharmacopeia 24, The National Formulary 19 United States Pharmacopeial Convention, Inc. Rockville; 2008.

33. Rosendorff C, Dubiel R, Xu J, Chavanu KJ: Comparison of olmesartan medoxomil versus amlodipine besylate on regression of ventricular and vascular hypertrophy. Am J Cardiol 2009, 3:359-65.

34. Chrysant SG: Amlodipine besylate/olmesartan medoximil fixed combination for the treatment of hypertension. Expert Rev Cardiovasc Ther 2009, 8:887-95.

35. Kereiakes DJ, Neutel JM, Punzi HA, Xu J, Lipka L, Dubiel R: Efficacy and safety of olmesartan medoxomil and hydrochlorothiazide compared with benazepril and amlodipine besylate. Am J Cardiovasc Drugs 2007, 5:361-72.

36. Chrysant SG, Weber MA, Wang AC, Hinman DJ: Evaluation of antihypertensive therapy with the combination of olmesartan medoxomil and hydrochlorothiazide. Am J Hypertens 2004, 3:252-9.

37. Deppeler HP: Hydrochlorothiazide. In Analytical profiles of drug substances. Volume 10. Edited by: Florey K. New York: Academic Press; 1981:405-441.

doi:10.1186/1752-153X-6-1

Cite this article as: Darwish et al:: Development of a novel 96-microwell assay with high throughput for determination of olmesartan medoxomil in its tablets. Chemistry Central Journal 2012 6:1.

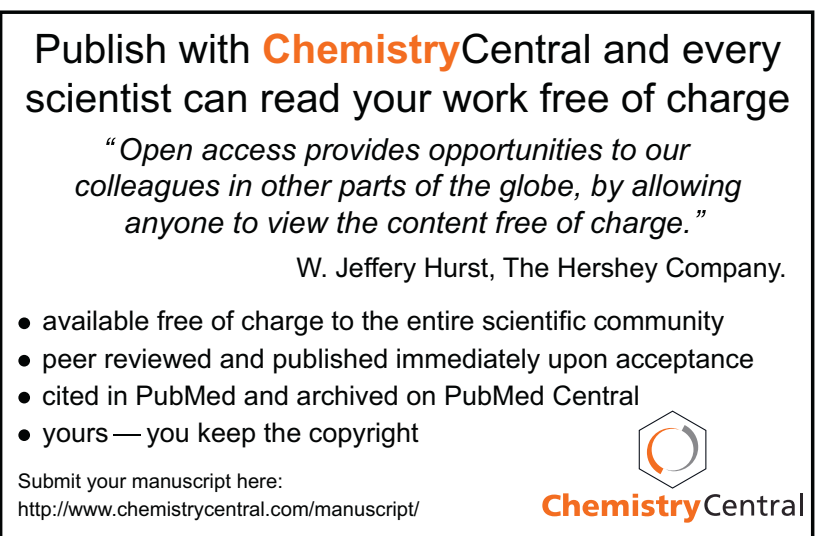

